\title{
Autoimmune Thyroiditis in Women of Child Bearing Age and its Association with Thyroid Function and Thyroid Antibody Status
}

\author{
${ }^{1}$ Shakila Zaman Rima, ${ }^{1}$ S M Moinul Islam, ${ }^{1}$ Nazmun Nahar, ${ }^{1}$ Rawshan Ara, ${ }^{1}$ Ratan Kumar Chakraborty, \\ ${ }^{1}$ Nasim Khan, ${ }^{1}$ Gazi Abul Hossain, ${ }^{1}$ Saiyeeda Mahmood, ${ }^{2}$ Mizanul Hasan \\ ${ }^{1}$ Institute of Nuclear Medicine and Allied Sciences, Mymensingh. \\ ${ }^{2}$ National Institute of Nuclear Medicine and Allied Sciences, Dhaka.
}

Correspondence: Dr. Shakila Zaman Rima, Senior Medical Officer, Institute of Nuclear Medicine\& Allied Seiences (INMAS), Mymensingh, P.O. Box. 47, Mymensingh, Bangladesh.

Mobile No:01558317740,E-mail: shakilarima17@gmail.com

\begin{abstract}
Thyroid disorders are prevalent in women of childbearing age and for this reason commonly present in pregnancy and the puerperium. Chronic autoimmune thyroiditis (AIT) is the most common and extensively studied organ-specific autoimmune disorder and it has important consequences on fertility, the occurrence of pregnancy complications and pregnancy outcome. Across sectional analytical study has been carried out on autoimmune thyroiditisamong the women of child bearing age with suspected thyroid disorders in the Centre for Nuclear Medicine \& Ultrasound, Mymensingh during the period of July, 2011 to June, 2012.The objectives of the study were to estimate the prevalence of autoimmune thyroiditis in women of child bearing age and to determine the association of autoimmune thyroiditis with thyroid function and thyroid antibody status. In the present study, 100 women of childbearing age (15-45Yrs) with any sorts of suspected thyroid disorders were included. But already diagnosed all follow up cases were excluded from the study. High Resolution Ultrasonography (HRUS) had been taken as principal modality of diagnosis of autoimmune thyroiditis. $T_{3}, T_{4}$ and TSH were taken as an important indicator for thyroid function status. Serum TM-Ab (Thyroid Microsomal Antibody) level and serum Tg-Ab (Thyroglobulin Antibody) level were measured to see the thyroid antibody status. In the present study, prevalence of autoimmune thyroiditis among the women of childbearing age with suspected thyroid disorders was found $41 \%$. According to the thyroid function status, $65.85 \%$ AIT patients were found in hypothyroid state, $29.27 \%$ in subclinical hypothyroid state and rest $4.88 \%$ were found in euthyroid state. In consideration of thyroid antibody status, 69.23\% AIT patients were positive \& $30.77 \%$ were negative for thyroid autoantibody. This study revealed that there were significant association of AIT with the biochemical hypothyroid state and positive thyroid antibody status.
\end{abstract}

Key Words: Autoimmune thyroiditis, Women of Child Bearing Age, Thyroid Function, Thyroid Antibody Status. 


\section{INTRODUCTION}

Autoimmune diseases are the leading causes of death among young and middle-aged women in the United States (1). According to the U.S. Department of Health and Human services, Office on Women's Health (2010), autoimmune diseases can affect anyone. Yet certain people are at greater risk, including women of childbearing age. More women than men have autoimmune diseases, which often start during their childbearing years (2).

Autoimmune thyroiditis or chronic autoimmune thyroiditis is a disease in which the body interprets the thyroid gland and its hormone products $\left(\mathrm{T}_{3}\right.$ and $\left.\mathrm{T}_{4}\right)$ and Thyroid Stimulating Hormone (TSH) as threats and produce special antibodies that target the thyroid cells and destroy it. It presents with hypothyroidism or hyperthyroidism and the presence or absence of goiters. If goiters are present, it is understood as Hashimoto's Thyroiditis. On the other hand, if the thyroid is atrophic, it is denominated Atrophic Thyroiditis. If the symptoms of thyroiditis appear in women after parturition, it is referred to as Postpartum Thyroiditis. Silent Thyroiditis and Postpartum Thyroiditis are now believed to be manifestations of chronic autoimmune thyroiditis (3).

In developed countries, chronic autoimmune thyroiditis is the most common cause of hypothyroidism among women in their childbearing age. Antibodies responsible for compromising maternal thyroid function can cross the placenta and can compromise fetal and neonatal thyroid function. Hypothyroidism in pregnant women can adversely affect their children's subsequent neuropsychological performance. Decreases in performance can occur even when the pregnant woman's hypothyroidism is mild and probably asymptomatic (4).

Uncorrected thyroid dysfunction in pregnancy has adverse effects on fetal and maternal well-being. The deleterious effects of thyroid dysfunction can also extend beyond pregnancy and delivery to affect neuro intellectual development in the early life of the child. Demand for thyroid hormones is increased during pregnancy, which may cause a previously unnoticed thyroid disorder to worsen (5).

Low thyroid reserve due to autoimmune thyroiditis is increasingly recognized as a serious health problem: 1) Thyroid autoimmunity increases the probability of spontaneous fetal loss. 2) Thyroid failure due to autoimmune thyroiditis, often mild and subclinical, can lead to permanent and significant impairment in neuropsychological performance of the offspring. 3) Evidence is emerging that as women age with sub clinical hypothyroidism, as a sequel of post partum thyroiditis, predisposes them to cardiovascular disorder. 
The prevalence of post partum thyroiditis ranges from 5-7\%. Postpartum thyroiditis is an acute stage of autoimmune thyroid destruction with subsequent repair in the context of an existing and ongoing process of thyroid auto-sensitization. This process frequently leads to a gradual development of permanent thyroid failure. The recognition of the true nature of this process, combined with recently described detrimental effects of a low thyroid reserve due to autoimmune thyroiditis, has important repercussions on clinical practice especially in women of childbearing age (6).

Benoist, et al. stated that more than 1.9 billion people of all ages were iodine deficient in the world; of them prevalence was lowest (10\%) in the Americas and highest (57\%) in the Europe and less than fifty percent (40\%) in South East Asia (7).

Iodine deficiency was found to be hyper-endemic in Bangladesh in 1993 in the national survey on goitre. Some subsequent investigations in the country attributed endemic goitre to widespread iodine deficiency. A few recent studies have observed the frequency of thyroid autoimmunity and cancer to be higher than it was before iodine prophylaxis in some countries. High iodine intake, as well as widely varying concentration of dietary iodine, day-to-day increases vulnerability of developing thyroid autoimmunity (8).

The study on prevalence of antithyroid microsomal antibody in thyroid patients of endemic goitre area by Mahmood, et al. at CNMU, Mymensingh revealed that the great majority (89.28\%) of the study subjects (both male \& female) had Hashimoto's thyroiditis or generalized features of autoimmune thyroid disorder (AITD). Among the total study population, female rated higher (59\%) with anti-microsomal antibody (TM-Ab) and highest number of cases ( $>90 \%)$ were found in 30-35 yrs of age group (9).

Autoimmune thyroiditis in women of childbearing age is such a clinical condition, which needs special attention. The concept of post partum thyroiditis being a mild and transient disorder is now changing. The recognition of the true nature of postpartum thyroiditis that is an acute phase in an ongoing and chronic thyroid autoimmune process, and the negative consequences of such a chronic process for offspring of affected mothers and for the mothers themselves, in the long run, are reasons to consider screening. 
In context of Bangladesh, very few data \& research findings are available about this health problem. So considering all these facts the present research work had been done.

The general objective of the study was to estimate the prevalence of Autoimmune Thyroiditis among the women of child bearing age with suspected thyroid disorders and to determine the association of Autoimmune Thyroiditis with thyroid function and thyroid antibody status.

The specific Objectives were:

1. To perform the High Resolution Ultrasound (HRUS) of thyroid glands of the study subjects to diagnose Autoimmune Thyroiditis.

2. To estimate the prevalence of Autoimmune Thyroiditis among the women of child bearing age with suspected thyroid disorders.

3. To measure the thyroid hormone profiles and thyroid autoantibody levels among the study subjects.

4. To establish the association of Autoimmune Thyroiditis with thyroid function status and thyroid antibody status.

\section{MATERIALS AND METHODS:}

This cross sectional analytical study was carried out for one year starting from July, 2011 to June, 2012in Centre for Nuclear Medicine \& Ultrasound (CNMU), Mymensingh. Female patients of childbearing age with suspected thyroid disorders, attending Centre for nuclear medicine \& Ultrasound, Mymensingh.

Women of childbearing age (15-45 yrs) having any sorts of suspected thyroid disorders were included. But already diagnosed or follow up cases of any thyroid disorders were excluded from the study to avoid repetition.

Non-random, purposive sampling included total 100 subjects.

All relevant data collected during the investigations were recorded on a pre-designed data collection sheet for each patient. Enlarged, diffusely hypoechoic, inhomogeneous tissue echotexture with or without internal septations on HRUS of thyroid gland is the basis of diagnosis of Autoimmune Thyroiditis.HRUS were done using ACCUVIX V 10, Medison, Korea with 5 to $13 \mathrm{MHz}$ linear array transducer \& Nemio 10, Toshiba, Medical system, Tokyo, Japan with 7 to $11 \mathrm{MHz}$ linear array transducer. 
Serum $\mathrm{T}_{3}, \mathrm{~T}_{4}$ and TSH were done in all patients. Serum $\mathrm{T}_{3}$ and $\mathrm{T}_{4}$ were done by Radioimmunoassay (RIA) and serum TSH was done by Immunoradiometric assay (IRMA). Estimation of serum thyroid microsomal antibody (TM-Ab) and thyroglobulin antibody (Tg-Ab) were done in all study subjects by RIA.

The collected data were compiled and subjected to relevant statistical analyses through the SPSS program. Chi- square tests were done to establish the association of AIT with other findings. The results of all tests were considered significant at $\mathrm{p}<0.05$.

Ethical consideration: The nature \& purpose of the study was such that no physical, psychological, social or legal risk was likely to be posed to the subjects. Subjects were inquired about relevant topics only to supplement the recorded data. The aims \& objectives of the study were explained to the patients, consents were acquired \& patients were assured that all information $\&$ records will be kept confidential.

\section{RESULTS}

Table 1: Number and percentage distribution of the patients according to the diagnosis based on HRUS (n=100)

\begin{tabular}{ccc}
\hline Diagnosis & $\begin{array}{c}\text { Number of patients } \\
\text { (n) }\end{array}$ & $\begin{array}{c}\text { Percentage } \\
(\%)\end{array}$ \\
\hline AIT & 41 & 41 \\
Non-AIT & 59 & 59 \\
\hline Total & $\mathbf{1 0 0}$ & $\mathbf{1 0 0}$ \\
\hline
\end{tabular}

According to the diagnosis based on HRUS findings, the patients were categorized as AIT and NonAIT. 41\% patients were diagnosed as AIT and 59\% patients were diagnosed as Non-AIT.

Table 2: Number \& percentage distribution of AIT patients according to the thyroid function status $(n=41)$

\begin{tabular}{ccc}
\hline Thyroid Function Status & Number of patients & Percentage (\%) \\
\hline Hypothyroid & 27 & 65.85 \\
Subclinical Hypothyroid & 12 & 29.27 \\
Euthyroid & 2 & 4.88 \\
Subclinical Hyperthyroid & 0 & 0.00 \\
Hyperthyroid & 0 & 0.00 \\
\hline Total & $\mathbf{4 1}$ & $\mathbf{1 0 0 . 0 0}$ \\
\hline
\end{tabular}


In this study, among the 41\% AIT patients, 27(65.85\%) patients presented in overt hypothyroid state, $12(29.27 \%)$ patients in subclinical hypothyroid state and $2(4.88 \%)$ patients presented in euthyroid state. No AIT patients were found in hyperthyroid or subclinical hyperthyroid state.

Table 3: Association of AIT with biochemical hypothyroid state (n=100)

\begin{tabular}{ccccc}
\hline $\begin{array}{c}\text { Hypothyroid state } \\
\text { (Hypothyroid+Subclinical } \\
\text { Hypothyroid) }\end{array}$ & $\begin{array}{c}\text { AIT } \\
\mathbf{n}(\%)\end{array}$ & $\begin{array}{c}\text { Non-AIT } \\
\mathbf{n}(\boldsymbol{\%})\end{array}$ & $\begin{array}{c}\text { Total } \\
\mathbf{n}(\%)\end{array}$ & p-Value \\
\hline Present & $39(39 \%)$ & $11(11 \%)$ & $50(50 \%)$ & $<\mathbf{0 . 0 0 1}$ \\
Absent & $2(2 \%)$ & $48(48 \%)$ & $50(50 \%)$ & \\
\hline Total & $\mathbf{4 1 ( 4 1 \% )}$ & $\mathbf{5 9 ( 5 9 \% )}$ & $\mathbf{1 0 0 ( 1 0 0 \% )}$ & \\
\hline
\end{tabular}

* Chi-Square test was done to measure the level of significance.

In this study, 39(39\%) AIT patients and 11(11\%) Non-AIT patients were in hypothyroid state. ChiSquare test demonstrated a significant association of AIT with the biochemical hypothyroid state $(\mathrm{p}<0.001)$.

Table 4: Number \& percentage distribution of AIT patients according to the thyroid antibody status $(n=39)$

\section{Thyroid Antibody Status $\quad$ Number of patients $\quad$ Percentage (\%)}

\begin{tabular}{ccc}
\hline Positive & 27 & 69.23 \\
Negative & 12 & 30.77 \\
\hline Total & $\mathbf{3 9}$ & $\mathbf{1 0 0 . 0 0}$ \\
\hline
\end{tabular}

Among the AIT patients, 27(69.23\%) patients were thyroid autoantibody positive and $12(30.77 \%)$ patients were negative.

Table 5: Association of AIT with positive thyroid antibody status (n=96)

\begin{tabular}{ccccc}
\hline $\begin{array}{c}\text { Thyroid Antibody } \\
\text { Status }\end{array}$ & $\begin{array}{c}\text { AIT } \\
\text { n (\%) }\end{array}$ & $\begin{array}{c}\text { Non-AIT } \\
\text { n (\%) }\end{array}$ & $\begin{array}{c}\text { Total } \\
\text { n (\%) }\end{array}$ & p-Value \\
\hline Positive & $27(28.13 \%)$ & $13(13.54 \%)$ & $40(41.67 \%)$ & \\
Negative & $12(12.50 \%)$ & $44(45.83 \%)$ & $56(58.33 \%)$ & $<\mathbf{0 . 0 0 1}$ \\
\hline Total & $\mathbf{3 9 ( 4 0 . 6 3 \% )}$ & $\mathbf{5 7 ( 5 9 . 3 7 \% )}$ & $\mathbf{9 6}(\mathbf{1 0 0 . 0 0 \% )}$ & \\
\hline
\end{tabular}

* Chi-Square test was done to measure the level of significance.

The result showed that among the 100 thyroid disordered patients, 96 were investigated for the thyroid antibody status. In case of 4 patients, thyroid antibody could not be done due to missing. Among the AIT patients, 27(28.13\%) were thyroid autoantibody positive and $12(12.5 \%)$ were 
thyroid autoantibody negative. And among the Non-AIT patients, 13(13.54\%) were thyroid autoantibody positive and 44(45.83\%) were thyroid autoantibody negative. Chi-square test demonstrated a significant association of AIT with the positive thyroid antibody status $(p<0.001)$.

\section{DISCUSSION:}

In the present study HRUS had been taken as principal modality for diagnosis of autoimmune thyroiditis based on several studies $(10,11,12,13,14)$. Normal thyroid shows homogenous echotexture and the level of echogenicity is more than that of surrounding muscles. Presence of diffuse hypoechoic, inhomogenousechotexture that is almost equal to or less than that of surrounding muscles, multiple small irregular echopenic, and linear echogenic areas and irregularity of thyroid borders are seen in autoimmune thyroiditis (10). Estimates of the prevalence of thyroid dysfunction depend upon methodological factors, classifications of hypothyroidism, geographic and environmental factors including iodine intake status and composition of the community examined by age, ethnicity and gender, making comparisons between studies of limited value (15).

In a countrywide screening among the healthy young girls in post iodization phase in India, the overall prevalence of Juvenile Autoimmune Thyroiditis (JAT) was 2.1\%, of which Hashimoto's Thyroiditis (HT) was 1.6\% and Focal Lymphocytic Thyroiditis (FLT) was 0.56\%; and among the goitrous girls, JAT was 7.5\% which included HT in 5.6\% and FLT in $1.9 \%$ respectively (16). The overall prevalence of JAT in 2801 schoolgirls surveyed by Jaksic, et al. (1994) was 0.6\%, whereas the goiter-specific prevalence was $12.4 \%$ (17). In a previous study on autoimmune thyroiditis among the puberty onset goitre based on HRUS by Chakraborty (2006) in CNMU, Mymensingh, the prevalence of autoimmune thyroiditis was found 36\% (18).In the present study, the prevalence of AIT among the women of child bearing age with suspected thyroid disorders was $41.0 \%$ on the basis of HRUS.

In a study on autoimmune thyroiditis in young girls in India, Marwaha, et al. (2000) found that $5.2 \%$ and $13.8 \%$ of girls with FNAC proven JAT had overt and sub-clinical hypothyroidism, respectively; $5.9 \%$ and $15.7 \%$ of girls who were diagnosed as JAT on serological criteria, had overt hypothyroidism and sub-clinical hypothyroidism respectively (16).

In a study on prevalence of thyroid microsomal antibody (TM-Ab) in thyroid patients in CNMU, Mymensingh, Mahmood, et al. (2004) found that thyroid antimicrosomal antibody (TM-Ab) was positive in $89.28 \%$ patients who had sonographic features of Hashimoto's thyroiditis or generalized 
feature of AIT in HRUS with hypothyroid state. TM-Ab was found positive in $33.33 \%$ of patients who had HRUS features of AIT with euthyroid state (9).

In another study in CNMU, Mymensingh by Chakraborty (2006) on autoimmune thyroiditis among the puberty onset goitre found that among the total 36 AIT patients, 19 patients $(52.78 \%)$ were hypothyroid, 8 patients $(22.22 \%)$ were subclinical hypothyroid and $9(25 \%)$ patients were biochemically euthyroid. None of the AIT patients were found in hyperthyroid or subclinical hyperthyroid state (18).

In the present study, according to the thyroid function status, among the total 41 AIT patients, 27 patients $(65.85 \%)$ were hypothyroid and 12 patients $(29.27 \%)$ were sub-clinical hypothyroid. Only 2 AIT patients (4.88\%) were euthyroid and no AIT patients were found in hyperthyroid or subclinical hyperthyroid state. This study revealed that there was a significant association of AIT with biochemical hypothyroid state $(\mathrm{p}<0.001)$.

Similar result was found in a study by Marwaha, et al. (2000) in India among the young girls, where none of the patients with HT had either overt hyperthyroidism or subclinical hyperthyroidism (16). Aryal, et al. (2010) in Kathmandu University Hospital, Nepal studied with 825 subjects; the prevalence of thyroid dysfunction was $25 \%$. Females had more thyroid dysfunction than males. Hypothyroidism (8\%) and subclinical hypothyroidism $(8 \%)$ had higher prevalence compared to subclinical hyperthyroidism (6\%) and hyperthyroidism (3\%) (15). In a countrywide screening of goitrous healthy young girls in India, by Marwaha, et al. (2000), it was found that among the girls with HT, both TM-Ab and Tg-Ab were positive in 39.5\%, TM-Ab alone in $67.4 \%$ and $\mathrm{Tg}-\mathrm{Ab}$ alone in $41.8 \%$ cases. And among the girls with FLT, individually TM-Ab and Tg-Ab were positive in $33.3 \%$ cases respectively and together in $28.5 \%$ cases of FLT. However in goitrous girls without autoimmune thyroiditis, $\mathrm{TM}-\mathrm{Ab}$ was positive in $8.7 \%$ and $\mathrm{Tg}-\mathrm{Ab}$ in $7.3 \%$ cases (16).

In a study on status of antithyroid antibodies in thyroid patients in Bangladesh by Hasanat, et al. (2000) found that among the clinically suspected AITD patients, 63.0\% with Hashimoto's thyroiditis and 44.7\% with atrophic thyroiditis were positive for antithyroid antibodies (8).I n a study on subclinical hypothyroidism and autoimmune thyroiditis in pregnancy in South Indian subjects, TPO antibodies were positive in $8.5 \%$ of the study subjects. Among the 14 women with hypothyroidism, 8(57.1\%) were TPO antibody positive and among the 481euthyroid women, 34(7.1\%) had TPO antibody positive (19). In the study in CNMU, Mymensingh on the the prevalence of anithyroid microsomal antibody in thyroid patients of endemic goitre area in 
Bangladesh by Mahmood, et al. found that among the total 221 patients, 126(57.01\%) patients were positive for $\mathrm{TM}-\mathrm{Ab}$. Among the all female thyroid patients, 59\% were positive for TM-Ab, whereas $51.67 \%$ of all male thyroid patients were positive. Highest $89.28 \%$ patients with sonographic features of AIT with hypothyroidism were found positive for TM-Ab (9). In another study on autoimmune thyroiditis among the puberty onset goitre in CNMU, Mymensingh by Chakraborty (2006), found that among the total 36 AIT patients $29(80.6 \%)$ were thyroid antibody positive and 7 (19.4\%) were thyroid antibody negative (18).

In the present study, out of total 39 AIT patients, $27(69.23 \%)$ patients were positive for thyroid autoantibody and $12(30.77 \%)$ patients were negative for thyroid autoantibody. There was a significant association of AIT with the positive thyroid antibody status $(\mathrm{p}<0.001)$.

\section{CONCLUSION}

In conclusion, this study revealed that the prevalence of AIT among the women of childbearing age with suspected thyroid disorders was $41 \%$ and AIT was associated with biochemical hypothyroid state and positive thyroid antibody status.

In comparison to other diseases for which screening is recommended, $41 \%$ prevalence of AIT among the women of child bearing age with suspected thyroid disorders seems to be high enough to justify screening in women of childbearing age.

Further population based epidemiological studies are needed to establish the accurate prevalence and predominant etiological factors of thyroid dysfunction in this region of the country. The study recommends screening in the population of women of child bearing age for autoimmune thyroiditis by HRUS with thyroid dysfunction \& thyroid autoantibody status to ensure early diagnosis and treatment and thereby to reduce the burden of the disease.

\section{REFERENCES:}

1. Cooper GS and Stroehla BC. The epidemiology of autoimmune diseases 2003.Autoimmunity Reviews; 2(3):119-125.

2.U.S. Department of Health and Human Services, Office on Women's Health. Autoimmune Diseases: Overview. Department of Health and Human Services, U.S.A.2010.[Online]Available at: http://www.womenshealth.gov[Accessed 15 may, 2012]

3. Dayan CM and Daniels GH. Chronic Autoimmune Thyroiditis. The New England Journal of Medicine1996; 335(2): 99-107.

4. Haddow JE, Palomaki GE, Allan WC, Williams JR, Knight GJ, Gagnon J, O’Heir CE, Mitchell ML, Hermos RJ, Waisbren SE, Faix JD and Klein RZ. Maternal thyroid deficiency during pregnancy and 
subsequent neuropsychological development of the child. The New England Journal of Medicine1999; 341(8): 549-555.

5.Vissenberg R, van den Boogaard E, van Wely M, van der Post JA, Fliers E, Bisschop PH and Goddijn M. Treatment of thyroid disorders before conception and in early pregnancy: a systematic review. Human Reproduction Update2012; 18(4): 360-373.

6. Muller AF, Drexhage HA and Berghout A. Postpartum Thyroiditis and Autoimmune Thyroiditis in Women of Childbearing Age: Recent Insights and Consequences for Antenatal and Postnatal Care.Endocrine Reviews2001;22(5): 605-630.

7. Benoist DB, Andersson MA, Takkouche B and Egli I. Iodine deficiency continues to be a major health problem worldwide. Clinical Thyroidology2004; 16(1): 18.

8. Hasanat MA, Rumi MAK, Alam MN, Hasan KN, Salimullah M, Salam MA, Fariduddin M, Mahtab H and Khan AKA. Status of antithyroid antibodies in Bangladesh. Postgraduate Medical Journal 2000; 76(896): 345-349.

9. Mahmood S, Islam MM, Siddiqui NI, Hossain GA, Chakraborty RK, Akhter N and Meah T. Prevalence of antithyroid microsomal antibody in thyroid patients of endemic goitre area. Mymensingh Medical Journal2004; 13(1): 4-10.

10. Ligan S, Arslan N, Bolu E, Aksu A, Ozturk E, Dundar S, Bayhan H and Gundogan MA. The Diagnostic Value of Ultrasound in the Detection of Hashimoto's Thyroiditis: Comparison with Serum Antibody Levels and Cytology.Turkish Journal of Endocrinology and Metabolism 1999; 3:109-112.

11. Chen YW, Chao MC, Hsiao PJ, Chung YW and Jaw TS. Combined Imaging Diagnosis of Tc-99mPertechnetateThyroid Scintigraphy and Ultrasonography in Autoimmune Thyroiditis of Young Children: A case report. Annals of Nuclear Medicine and Sciences 2002; 15: 223-226.

12. Loy M, Cianchetti ME, Cardia F, Melis A, Boi F and Mariotti S. Correlation of computerized gray-scale sonographic findings with thyroid function and thyroid autoimmune activity in patients with Hashimoto's thyroiditis. Journal of Clinical Ultrasound2004; 32 (3): 136-140.

13. Gartner R. Modern diagnostic approach to autoimmune thyroiditis. MMW Fortschritte der Medizin 2006; 148(16): 41-45.

14. Zois C, Stavron I, Svarna E, Seferiadis K and Tsatsoulis A. Natural course of autoimmune thyroiditis after elimination of iodine deficiency in northwestern Greece. Thyroid 2006; 16(30): 289-293.

15. Aryal M, Gyawali P, Rajbhandari N, Aryal P and Pandeya DR.A prevalence of thyroid dysfunction in Kathmandu University Hospital, Nepal. Biomedical Research 2010;21(4): 411-415.

16. Marwaha RK, Tandon N, Karak AK, Gupta N, Verma K and Kochupillai N.Hashimoto's Thyroiditis: Countrywide Screening of Goitrous Healthy Young Girls in Postiodization Phase in India. The Journal of Clinical Endocrinology \& Metabolism 2000; 85(10): 3798-3802.

17. Jaksic J, Dumic M, Filipovic B, IlleJ, Cvijetic M andGjuric G. Thyroid diseases in a school population with thyromegaly. Archives of Disease in Childhood 1994; 70(2): 103-106. 
18. Chakraborty RK. Autoimmune thyroiditis among puberty onset goiter based on High Resolution Ultrasound. MPhil 2006. Institute of Nuclear Medicine \&Ultrasound, Bangabandhu Sheikh Mujib Medical University.

19. Gayathri R, Lavanya S and Raghavan K. Subclinical Hypothyroidism and Autoimmune Thyroiditis in Pregnancy-A Study in South Indian Subjects. The Journal of the Association of Physicians of India 2009; 57: 691-693. 